\title{
ON THE GROUP OF AUTOMORPHISMS OF
}

\section{A TOPOLOGICAI GROUP*)}

\author{
By \\ Katsumi Nomizu and Morikuni Gotó
}

Let $G$ be a locally compact topological group and let $A(G)$ be the group of all (continuous) automorphisms of $G$. In $\S 1$ of the present note, we introduce a topology into $A(G)$ such that $A(G)$ becomes a topological group"). Our topology is a very natural one in view of the properties stated there. Our main concern, in the present note, will be then to investigate the condition for $A(G)$ to be compact with respect to this topology. For a connected Lie group, the problem is completely solved (\$2). But the situations are more complicated in the case of a general locally compact connected group and we can not yet obtain a satisfactory result. Some remarks and examples will be added in $\S 3$.

$\S 1$. Let $G$ be a locally compact group and $A(G)$ the group of all (continuous) automorphisms of $G$. Cosider the totality $\Sigma$ of pairs $\alpha=\left(K, V^{r}\right)$, where $K$ is, in each pair, a compact subset of $G$ and $V$ is a neighbourhood of the identity $e$ of $G$. If we define a partial order in $\Sigma$ such that, for $\alpha=(K, V)$ and $\alpha^{\prime}=\left(K^{\prime}, V^{\prime}\right), \alpha>\alpha^{\prime}$ means $K \supset K^{\prime}$ and $V \subset V^{\prime}, \Sigma$ becomes a directed set. For any $\alpha \in \Sigma, \alpha=(K, V)$, put

$$
U_{a}=\left\{\sigma \varepsilon A(G) ; x^{\sigma-1} \varepsilon V \text { and } x^{\sigma^{-1}-1} \varepsilon \forall \text { for all } x \in K\right\} \text {. }
$$

Clearly $U_{\alpha}=U_{a}^{-1}$ and $U_{\alpha} \subset U_{\alpha^{\prime}}$ if $\alpha>\alpha^{\prime}$ in $\Sigma$. It is easy to verify that, by taking the set of all $U_{\alpha}, \alpha \varepsilon \Sigma$ as a complete system of neighbourhoods of the identity, $A(G)$ becomes a topological group.

This topology of $A(G)$ has the following properties :

*) Received Sept. 8, 1949.

1) For a group of homeomorphisms of a topological space, see H. Nagao: On the Topologies of Homeomorphism Groups of Topological Spaces, Osaka Math. Journ. vol. 1. No 1 1949. Also, J. Dieudonné: On Topological Groups of Homeomorphisms, Amer, Journ, of Math, vol, LXX, No, 3 (1948), 
(1) $x^{\sigma}$ is continnous in $(x, \sigma) \varepsilon G \times A(G)$. Morever this topology is the weakest one among the topologies of $A(G)$ having this property.

(2) The natural homomorphism from $G$ into $A(G)$ letting correspond to an element $x \in G$ the inner automorphism of $G$ induced by $x$ is continuous. The subgroup $I(G)$ of all inner automorphisms is not necessarily closed in $A(G)$.

(3) If $G$ is moreover connected, we can define same topology of $A(G)$ by considering the system of $U(V)=U_{\alpha}, \bar{\alpha}=(W, V)$, where $W$ is an arbitrary but fixed neighbouhood of $e$ with compact closure.

(4) If $G$ is a connected Lie group, $A(G)$ is a Lie group as a linear group of automorphisms of the Lie algebra of $G$. The topology of this Lie group $A(G)$ coincides with.the one defined above.

(5) Let $G$ be a locally compact abelian group and $G^{*}$ the character group of $G$. The well known dual isomorphism?) of $A(G)$ and $A\left(G^{*}\right)$ is topolc g.cal if $A(G)$ and $A\left(G^{*}\right)$ are topologized as above.

\$2. In this $\S$ we shall prove the following.

THEOREM 1. Let $G$ be a connected Lie group. Then necessary and sufficient condition that $A(G)$ be compact is that $G$ is compact and bas a center of dimenssion less than?

ERoof. Necessity: Let $A(G)$ be compact. Then the adjojnt group $I(G)$ of $G$ is fully reduecible as a linear subgroup of the compact $A(G)$. From this we see that

$$
G=Z^{0} \cdot S, \quad\left[Z^{0}, S\right]=e,
$$

where $Z^{0}$ is the component of the identity of the center $Z$ of $G$ and $S$ is the maximal semi-simple normal subgroup of $G . G / Z$ is semi-simple as it is locally isomorphic with $G / Z^{0}$. Moreover $G / Z$ is maximaly almost periodic, for it is continuously isomorphic with $I(G)$ which is maximally almost periodic. Thus $G / Z$ is compact. Aa $S$ is semi-simple and locally isomorphic with compact $G / Z, S$ is also compact by a theorem of Weyl. As $Z^{0}=V \times T$ we have $G=(V \times T) S=V \times(T S)$, where $V$ is a vector group, $T$ is a toroidal group and $S$ is a semi-simple compact normal subgroup of $G$. But as $A(G)$ is compact, $G$ can not contain $V$. Hence $G=T S$. We must show that the dimension of $T$ is $\leqq$. If $\sigma$ is an automorphism of $T$ leaving invariant every element of $T \cap S$, we obtain an automorphism of $G$ by setting $g^{\sigma}=t^{\sigma} S$

2) E.g., M. Abe Uber Automorphismen der lokal-kompakten abelschen Gruppen, Proc, Imp. Acad, Tokyo, vol, 16 (1940) , 
for $g=t s, t \in T, s \in S$. Hence we can conclude our proof by the following lemma which is easy to prove.

Lemma. Let $T$ be a toroidal group of dimension $\geqq 2$, and let $F$ be any finite subgroup of $T$. Then there exist infinitely many automorphisms of $T$ which leave invariant every element of $F$.

Sufficiency: First, if $G$ is compact and semi-simple, $A(G)$ is clearly compact. Let $G=T S$, where $T$ is a one-dimensional central toroidal group and $S$ is s semi-simple normal subgroup of compact $G$. Then $A(G)$ is a subgroup of $A(T) \times A(S)$, which is compact. $I(G)$ is isomorphic with $I(S)$, which is known to be the component of the identity of $A(S)$. So $A(T) \times$ $A(S)^{\prime} I(S)$ is finite.

Theorem 1 is thus proved.

When $G$ is not a Lie group, we can prove, by a similar arguement as in the first half of Theorem 1 , the following

Theorem 2. Let $G$ be a locally compact connected group. If $A(G)$ is compact, then $G$ is also compact.

The proof is omitted.

\$3. We want to add some supplementary remarks and examples concerning the problem in the case of a compact connected abelian group. In the following $G$ will always denote such a group.

(1) As is rcmarked in $\S 1, A(G)$ is isomorphic with $A\left(G^{*}\right), G^{*}$ being the discrete character group of $G$. $G^{*}$ contains no element of finite order and the rank of $G^{*}$ is equal to the dimension of $G$. Hence, if $G$ is finite-dimensional. $G^{*}$ is a subgroup of the finite direct product of the discrete additive group $\mathrm{R}$ of rational numbers and $A(G)=A\left(G^{*}\right)$ is discrete.

(2) Let $\operatorname{dim} G=\operatorname{rank} G^{*}=1$. Then $G^{*}$ is a subgroup of $R$ and $A(G)=$ $A\left(G^{*}\right)$ is finite (of order 2) if and only if $G^{*}$ is isomorphic with the group of integers or $G^{*}$ satisfies the following condition :

In the irreduaible fractional expressions of the elements of $G^{*}$, every prime number $p$ appears in the denominators with bounded exponents.

(3) By (2) we see that, for any integer $n>0$, there exists an $n$-dimensional $G$ with finite $A(G)$. Such an example can be constructed as follows :

Divide the set of all prime numbers into $n$ disjoint classes $P_{k}(k=1,2, \cdots, n)$, each containing infinitely many prime numbers. Let $G_{k}^{*}(k=1,2, \cdot, n)$ be the subgroup of $\mathrm{R}$ consisting of rational numbers or the form $q^{\prime} p_{i 1} \cdots p_{i s}$, where $q$ is an integer and $p_{i 1}, \cdots, p_{i s}$ are any different prime numbers belonging to $P_{k}$. 
The character group $G$ of the direct product group of $G_{k}^{*}(k=1, ?, \cdots, n)$ is the one required.

In an analogous way, we can show the existence of an infinite-dimensional $G$ with compact $A(G)$.

(4) If $G$ contains a toroidal group ot any dimension as a proper direct factor, then $A(G)$ is not compact.

Mathematical Institute, Nagoya University, Nagoya. 